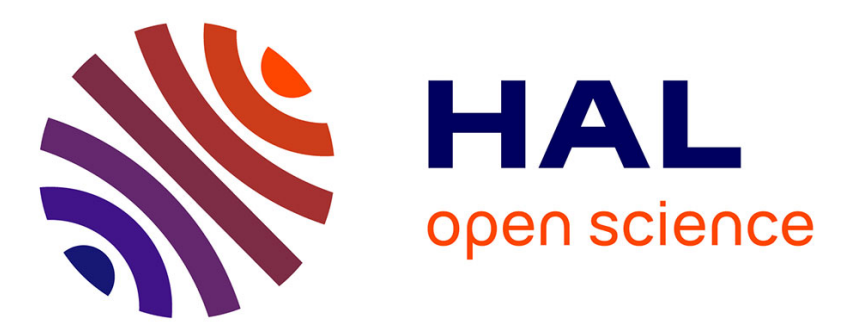

\title{
Experimental and constitutive modeling of a filled rubber with emphasis on the dynamical properties
}

\author{
Alexis Delattre, Stéphane Méo, Florian Lacroix, Caroline Richard, Stéphane
}

Lejeunes

\section{- To cite this version:}

Alexis Delattre, Stéphane Méo, Florian Lacroix, Caroline Richard, Stéphane Lejeunes. Experimental and constitutive modeling of a filled rubber with emphasis on the dynamical properties. ECCMR 2013, 2013, San Sebastian, Spain. pp.xx-xx. hal-00859662

\section{HAL Id: hal-00859662 \\ https://hal.science/hal-00859662}

Submitted on 10 Sep 2013

HAL is a multi-disciplinary open access archive for the deposit and dissemination of scientific research documents, whether they are published or not. The documents may come from teaching and research institutions in France or abroad, or from public or private research centers.
L'archive ouverte pluridisciplinaire HAL, est destinée au dépôt et à la diffusion de documents scientifiques de niveau recherche, publiés ou non, émanant des établissements d'enseignement et de recherche français ou étrangers, des laboratoires publics ou privés. 


\title{
Experimental and constitutive modeling of a filled rubber with emphasis on the dynamical properties
}

\author{
A. Delattre, S. Méo, F. Lacroix \& C. Richard \\ Laboratory of Mechanics and Rheology \\ Francois Rabelais University, Tours, France \\ S. Lejeunes \\ Laboratory of Mechanics and Acoustics \\ CNRS UPR 7051, Marseille, France
}

\begin{abstract}
This study relates to a project that aims to develop a specific numerical tool to simulate the behavior of elastomeric bearings or laminated devices used in helicopter rotors. In these applications, the elastomeric material can be filler-reinforced synthetic rubber or natural rubber. In normal flight conditions, such structures are submitted to multi-axial, multi-frequencies and multi-amplitudes loadings. Furthermore, the design of these parts has to take into account of a large range of temperatures $\left(-40^{\circ} \mathrm{C}\right.$ to $\left.70^{\circ} \mathrm{C}\right)$.

Here, characterization tests carried out on a carbon-black filled butadiene rubber are presented. The expremental campaign comprises static and dynamic tests at various controlled temperatures on uniaxial (tension, compression, simple shear and torsion) or multiaxial specimens (tension-torsion). The aim of this characterization is to determine the dynamical stabilized behavior (de-Mullinsized). As expected, the material exhibits a non-linear viscoelastic behavior. The Payne effect and the frequency dependency are observed in a range of amplitudes and frequencies corresponding to flight conditions. Multi-step relaxation tests show that the dissipation is not fully viscoelastic and that other dissipative phenomena take place in the material.

To model the material behavior, we investigate the reliability of three material models to predict uni- and multiaxial stabilized dynamic behavior. These models are all based on the thermodynamics of irreversible processes at finite strain. The first one is based on a statistical rheological approach and is mainly an extension of the model developed by Martinez et al. (Martinez, Boukamel, Méo, \& Lejeunes 2011). The second one is purely phenomenological: scalar internal variables associated with time-dependent effects are introduced and combined with a Zener model. The last one is the Linder et al. model (Linder, Tkachuk, \& Miehe 2011).
\end{abstract}

\section{INTRODUCTION}

For years, elastomers are widely used in industry. Their microstructure confers on them a dissipative behavior and a high deformability. These properties, amongst others, make these materials an appropriate candidate for many industrial applications. This study focuses particularly on elastomeric bearings and laminated devices used in helicopter rotors. For safety reasons, those parts are supposed to support extreme operating conditions, in terms of temperatures and loadings. Thus, to meet the specifications, their mechanical behavior must be well known when they are submitted to dynamic loading, with several frequencies, large amplitudes, on a wide range of temperatures.

To model the visco-elastic behavior of rubber-like materials, several approaches can be used:
- Models with integral formulation consider stress as a function of strain history (e.g. (Coleman \& Noll 1961)).

- Micro-physically motivated models are based on interactions between the fillers and the matrix (e.g. (Miehe \& Göktepe 2005)).

- Differential formulations use the concept of intermediate states (e.g. (Méo 2000)).

Hereafter, we choose a differential formulation to describe the material response to large strains and to several frequencies. The Payne effect and the frequency dependency are taken into account by the introduction of internal variables integrated in a model which is analoguous to a rheological assembly with several dissipative branches. 
In this paper, some results of the characterization campaign are presented. Amplitude- and frequencydependency are especially highlighted in both axial and shear loadings. Step-relaxation tests are also carried out to exhibit the static behavior of the material.

Then, we propose a new model, based on the thermodynamics of irreversible processes, to describe the material response. Last, the reliability of this model and two other ones issued from literature, is investigated by comparition with expermiental tests.

\section{EXPERIMENTAL OBSERVATIONS}

\subsection{Tests description}

In accordance with our industrial applications, we aim to characterize the multi-axial, dynamical, stabilised behavior of a carbon-black filled Butadiene Rubber in a frequency range from 0 to $30 \mathrm{~Hz}$, and for strain amplitudes up to $100 \%$. In order to do this, we carried out an experimental campaign on double-shear, $\mathrm{H} 2$ and diabolo specimens. These specimens are consistently softened before each test in order to eliminate the Mullins effect, that is not to be taken into account in our modeling. In details, this campaign relies on the following tests:

- Relaxation tests and step-relaxation tests.

- Quasi-static tests (ie at low strain rate).

- Cyclic tests at several amplitudes and several frequencies, with or without pre-strain.

- Physical chemistry characterization (DMA and DSC).

Moreover, these tests were carried out in a climate chamber at several temperatures $\left(-40^{\circ} \mathrm{C}\right.$ and $\left.70^{\circ} \mathrm{C}\right)$.

\subsection{Experimental results}

The Fig.1 shows us that the viscoelastic response of the material significantly depends on the strain amplitude. Indeed, the relaxation stress is higher for low strain levels. Moreover, the dissipative behavior of the material is mainly viscoelastic: in the Fig.2 and Fig.3, the relaxation stress in the loading phase and in the unloading phase are almost identical for a given deformation level. By joining those relaxed stress, the hyper-elastic behavior can be defined. This behavior represents the static response of the material. In these graphs, we exploit the relaxation stress in the unloading phase: in a visco-plastic case, the plastic stress is thereby eliminated. We obtain a rubber-like typical hyper-elastic behavior. The material presents a softening response for small strain amplitudes and then stiffer for larger amplitudes.

In this study, we focuses on the influence of strain amplitude and frequency on the stiffness and on the

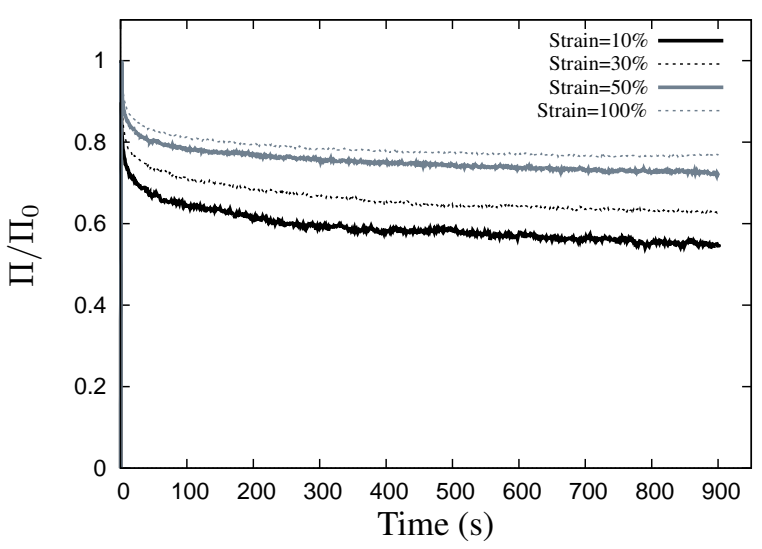

Figure 1: Normalized relaxation tests at several strain levels

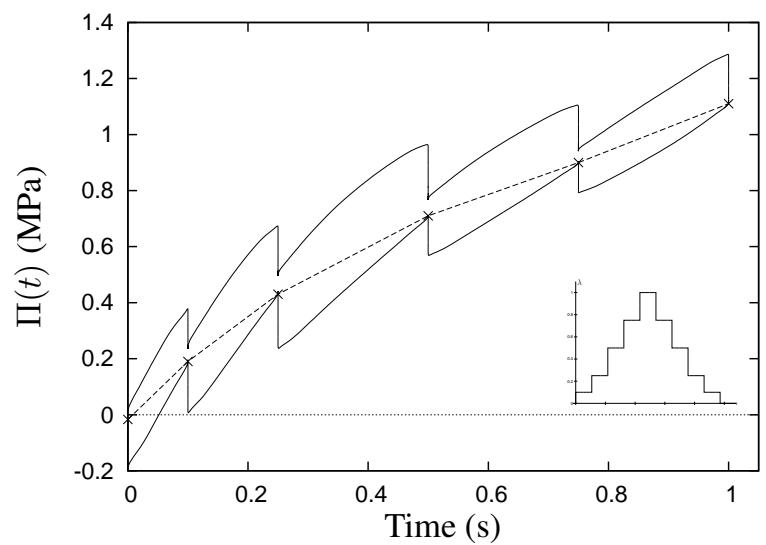

Figure 2: Step-relaxation in the case of tensile load

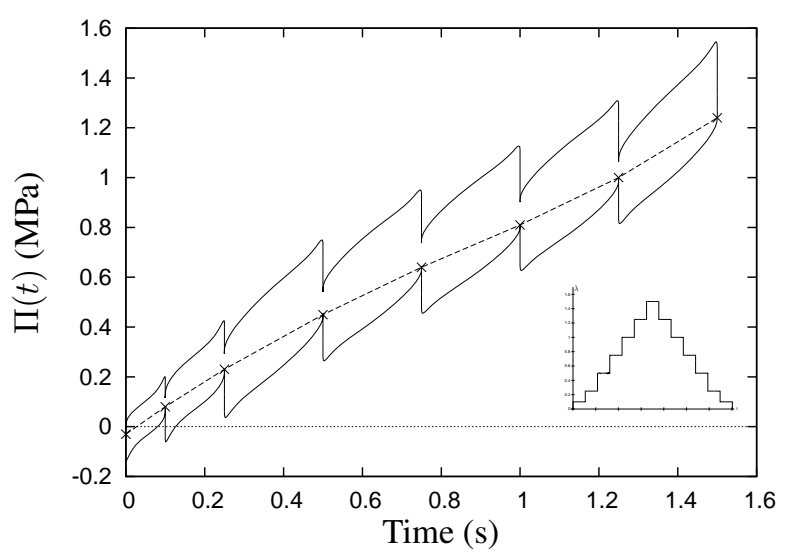

Figure 3: Step-relaxation in the case of shear load

dissipation. We define the stiffness as the slope of the straight line throught the hysteresis extrema, and we assume that the hysteresis area is directly associated to the dissipation per cycle. The curves Fig.4 represents these dependencies. The stiffness and the dissipation per cycle sligtly increase with frequency, but the Payne effect is much more pronounced in our strain range.

Lots of models and approaches can be found in the literature to describe visco-elastic behaviors with frequency dependencies, that give good correlations with experimental results (see e.g. (Höfer \& Lion 2009) and (Miehe \& Göktepe 2005)). However, amplitudedependent behavior at large strains is more rarely taken into account (see (Martinez 2005)) because this 


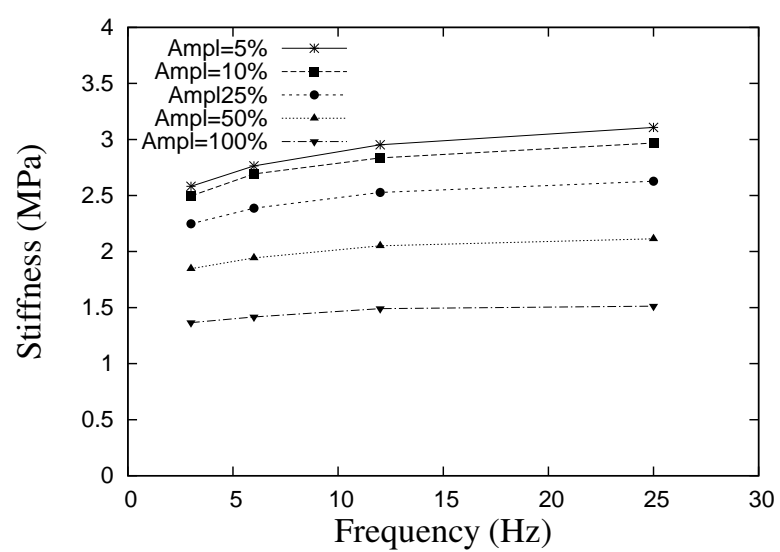

(a) Effect of frequency on dynamic stiffness

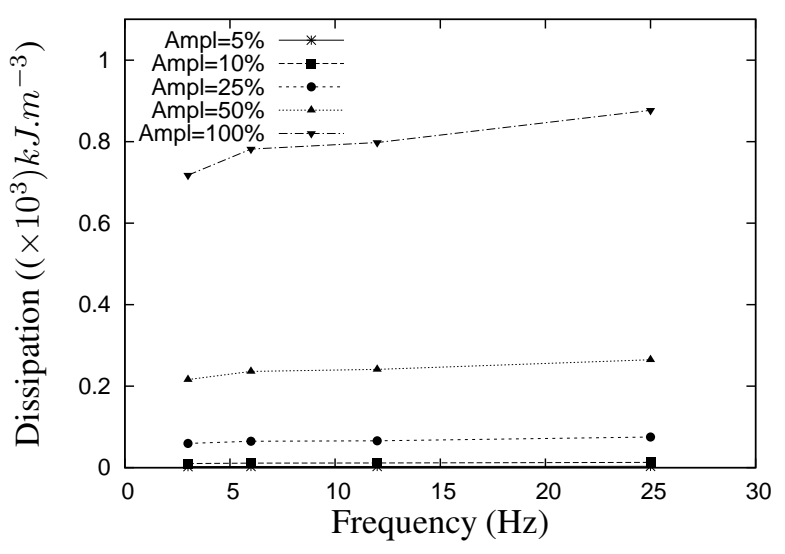

(c) Effect of frequency on dissipation

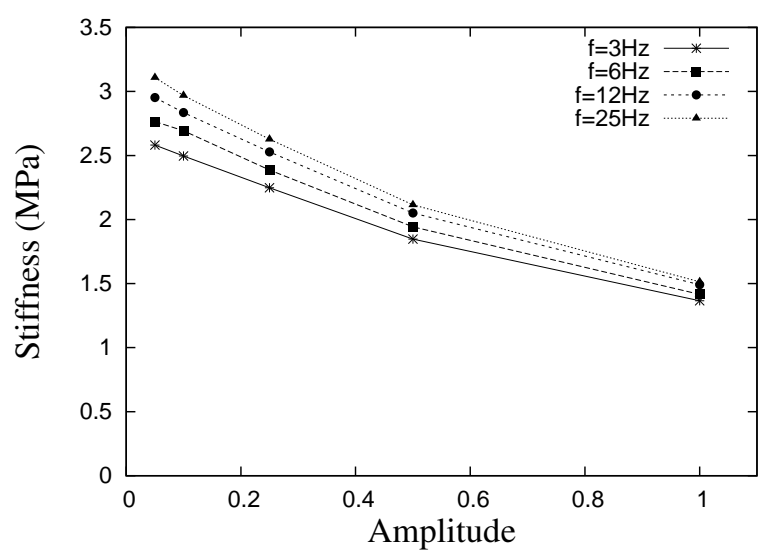

(b) Effect of amplitude on dynamic stiffness

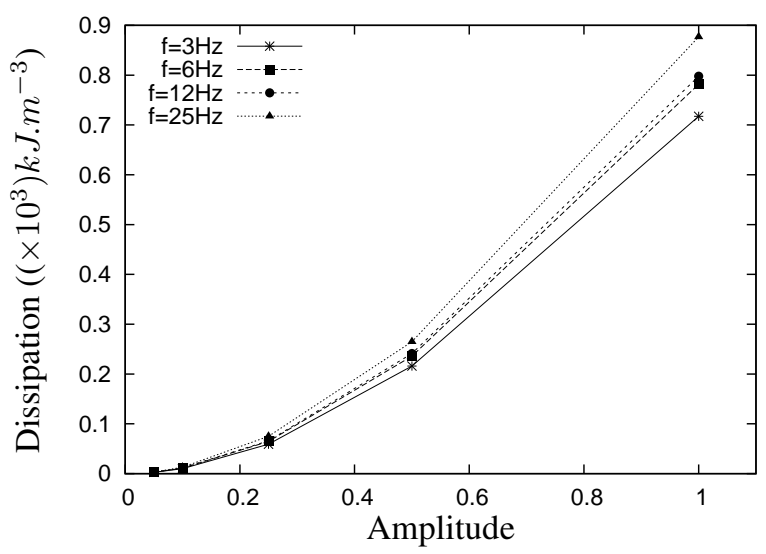

(d) Effect of amplitude on dissipation

Figure 4: Effects of frequency and strain amplitude on dynamic stiffness and dissipation during a cycle

reversible effect is not naturally integrated into classical models. As far as we know, there is no viscohyper-elastic approach describing the effects of both frequency and large amplitudes, and valid for multiaxial loadings.

\section{CONSTITUTIVE EQUATIONS}

\subsection{Thermodynamical setting}

As we consider the material as quasi-incompressible, the transformation gradient tensor $\mathbf{F}$ can be split into a volumic part and an isochoric part:

$\mathbf{F}=\left(J^{1 / 3} \mathbf{I d}\right) \cdot \overline{\mathbf{F}}$

Where Id is the second-order identity tensor.

Furthermore, by using the concept of intermediate state (see (Sidoroff 1974) and (Sidoroff 1975)), the isochoric part of the transformation gradient tensor is decomposed in an elastic part and a viscous part:

$\overline{\mathbf{F}}=\overline{\mathbf{F}}_{e} \cdot \overline{\mathbf{F}}_{v}$
Let $\boldsymbol{\sigma}$ be the Cauchy stress tensor, $\mathbf{D}$ the eulerian strain rate tensor and $J$ the determinant of $\mathbf{F}$. Neglicting the thermal effects, we assume that in the eulierian configuration, the Clausius-Duhem inequality can be written as follows:

$\phi=\boldsymbol{\sigma}: \mathbf{D}-\rho_{0} J^{-1} \dot{\psi} \geq 0$

Where $\phi$ is the intrinsic dissipation, $\rho_{0}$ is the density in the initial configuration.

Then, we choose to express the specific free energy as the sum:

$\psi\left(\overline{\mathbf{B}}, \overline{\mathbf{B}}_{e}, \omega, J\right)=\psi_{0}^{i s o}(\overline{\mathbf{B}})+\psi_{v}^{i s o}\left(\overline{\mathbf{B}}_{e}, \omega\right)+\psi^{v o l}(J)$

$\overline{\mathbf{B}}=\overline{\mathbf{F}} \cdot \overline{\mathbf{F}}^{T}$ and $\overline{\mathbf{B}}_{e}=\overline{\mathbf{F}}_{e} \cdot \overline{\mathbf{F}}_{e}^{T}$ are the isochoric left Cauchy-Green tensor and the elastic isochoric left Cauchy-Green tensor, respectively. $\omega \in[0,1]$ is an internal variable representing the breakdowns between the filler agglomerates and the polymer matrix. This phenomenom is at the origin of the Payne effect. It is a reversible process so that the filler-chain links can reform (see (Heinrich \& Klüppel 2002)). However, in the following, the reversible aspect won't be taken into account. 
We can therefore write $\dot{\psi}$ as:

$$
\begin{aligned}
\dot{\psi}\left(\overline{\mathbf{B}}, \overline{\mathbf{B}}_{e}, J\right)= & \frac{\partial \psi_{0}^{\text {iso }}(\overline{\mathbf{B}})}{\partial \overline{\mathbf{B}}}: \dot{\overline{\mathbf{B}}}+\frac{\partial \psi_{v}^{i s o}\left(\overline{\mathbf{B}}_{e}, \omega\right)}{\partial \overline{\mathbf{B}}_{e}}: \dot{\overline{\mathbf{B}}} \\
& +\frac{\partial \psi_{v}^{i s o}\left(\overline{\mathbf{B}}_{e}, \omega\right)}{\partial \omega} \dot{\omega}+\frac{\partial \psi^{v o l}(J)}{\partial J} \dot{J}
\end{aligned}
$$

With:

$$
\left\{\begin{array}{l}
\dot{\overline{\mathbf{B}}}=\mathbf{L} \cdot \overline{\mathbf{B}}+\overline{\mathbf{B}} \cdot \mathbf{L}^{T}-\frac{2}{3} \operatorname{Tr}(\mathbf{D}) \overline{\mathbf{B}} \\
\dot{\overline{\mathbf{B}}}_{e}=\mathbf{L} \cdot \overline{\mathbf{B}}_{e}+\overline{\mathbf{B}}_{e} \cdot \mathbf{L}^{T}-\frac{2}{3} \operatorname{Tr}(\mathbf{D}) \overline{\mathbf{B}}_{e}-2 \overline{\mathbf{V}}_{e} \cdot \overline{\mathbf{D}}_{v}^{o} \cdot \overline{\mathbf{V}}_{e} \\
\dot{J}=J \operatorname{Tr}(\mathbf{D})
\end{array}\right.
$$

Where $\overline{\mathbf{D}}_{v}^{o}=\frac{1}{2} \mathbf{R}_{e} \cdot\left(\dot{\overline{\mathbf{F}}}_{v} \cdot \overline{\mathbf{F}}_{v}^{-1}+\overline{\mathbf{F}}_{v}^{-T} \cdot \dot{\overline{\mathbf{F}}}_{v}^{T}\right) \cdot \mathbf{R}_{e}^{T}$ is an objective mesure of the isochoric viscous deformation rate and $\overline{\mathbf{V}}_{e}$ is a isochoric pure deformation tensor.

Let's assume that each term of the intrinsic dissipation (3) are independently positive, which is a usual assumption to ensure the Clausius-Duhem inequality to be verified. Moreover, making the hypothesis that each thermodynamical force obey a normality principle with respect to a dissipation potential $\varphi_{v}\left(\overline{\mathbf{D}}_{v}^{o}\right)$, equations (5), (6) and (3) are combined to obtain the following constitutive equation and evolution law:

$$
\left\{\begin{array}{l}
\boldsymbol{\sigma}=\boldsymbol{\sigma}_{0}+\boldsymbol{\sigma}_{v}+\rho_{0} \frac{\partial \psi^{v o l}(J)}{\partial J} \mathbf{I d} \\
\frac{\partial \varphi_{v}\left(\overline{\mathbf{D}}_{v}^{o}\right)}{\partial \overline{\mathbf{D}}_{v}^{o}}=2 \rho_{0} J^{-1}\left[\overline{\mathbf{V}}_{e} \cdot \frac{\partial \psi_{v}^{i s o}\left(\overline{\mathbf{B}}_{e}, \omega\right)}{\partial \overline{\mathbf{B}}_{e}} \cdot \overline{\mathbf{V}}_{e}\right]^{D} \\
-\rho_{0} J^{-1} \frac{\partial \psi_{v}^{i s o}\left(\overline{\mathbf{B}}_{e}, \omega\right)}{\partial \omega} \dot{\omega} \geq 0
\end{array}\right.
$$

With:

$$
\left\{\begin{array}{l}
\boldsymbol{\sigma}_{0}=2 \rho_{0} J^{-1}\left[\overline{\mathbf{B}} \cdot \frac{\partial \psi_{0}^{i s o}(\overline{\mathbf{B}})}{\partial \overline{\mathbf{B}}}\right]^{D} \\
\boldsymbol{\sigma}_{v}=2 \rho_{0} J^{-1}\left[\overline{\mathbf{B}}_{e} \cdot \frac{\partial \psi_{v}^{\text {iso }}\left(\overline{\mathbf{B}}_{e}, \omega\right)}{\partial \overline{\mathbf{B}}_{e}}\right]^{D}
\end{array}\right.
$$

The equation (7) can be seen as a rheologic analogy with a unique dissipative branch. This corresponds to the Zener model described in Fig.5.

\subsection{Behavior law setting-up: the various potentials}

The expression chosen for $\psi_{0}^{\text {iso }}(\overline{\mathbf{B}})$ is the form given in (Gornet, Macrkmann, Desmorat, \& Charrier 2012):

$$
\begin{aligned}
\psi_{0}^{\text {iso }}\left(I_{1}(\overline{\mathbf{B}}), I_{2}(\overline{\mathbf{B}})\right)= & c_{1} \int e^{\left[c_{3}\left(I_{1}(\overline{\mathbf{B}})-3\right)^{2}\right]} d I_{1} \\
& +3 c_{2} \int \frac{1}{\sqrt{I_{2}(\overline{\mathbf{B}})}} d I_{2}
\end{aligned}
$$

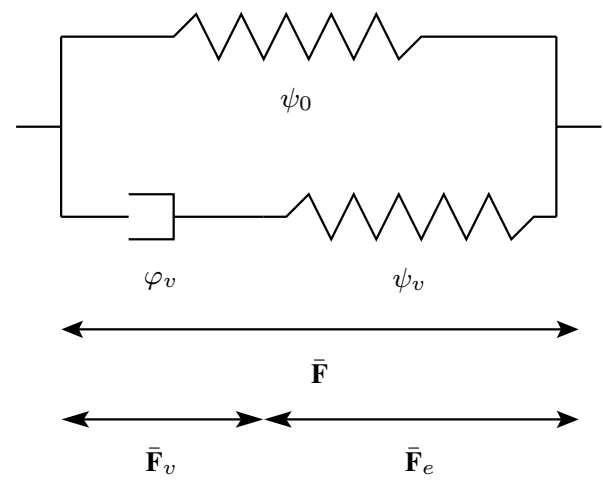

Figure 5: Zener model

Where $I_{1}(\overline{\mathbf{B}})$ and $I_{2}(\overline{\mathbf{B}})$ are the first and the second invariants of $\overline{\mathbf{B}}$, respectively, and $c_{1}, c_{2}$ and $c_{3}$ are material parameters.

Concerning the dissipative branch, a neo-hookean form is chosen for $\psi_{v}^{i s o}\left(\overline{\mathbf{B}}_{e}, \omega\right)$, with the coefficient $G(\omega)$ varying linearly with $\omega$. We choose a quadratic form for the pseudo-potential of viscous dissipation $\varphi_{v}\left(\overline{\mathbf{D}}_{v}^{o}\right)$. These potential can be written as follows:

$$
\left\{\begin{array}{c}
\psi_{v}^{i s o}\left(\overline{\mathbf{B}}_{e}, \omega\right)=G(\omega)\left(I_{1}\left(\overline{\mathbf{B}}_{e}\right)-3\right) \\
\text { with } G(\omega)=G_{0} \omega \\
\varphi_{v}\left(\overline{\mathbf{D}}_{v}^{o}\right)=\frac{\eta}{2} \overline{\mathbf{D}}_{v}^{o}: \overline{\mathbf{D}}_{v}^{o}
\end{array}\right.
$$

To define the evolution law for $\omega(t)$, we must keep in mind that the inequality $(7 \mathrm{c})$ must be verified. Noting that the equation (10a) makes $\frac{\partial \psi_{v}^{i s o}\left(\overline{\mathbf{B}}_{e}, \omega\right)}{\partial \omega}$ be always positive, $\dot{\omega}$ necessarily needs to be negative. We choose:

$\dot{\omega}=\frac{1}{h}\left(\left(\frac{3}{I_{1}(\overline{\mathbf{B}})}\right)^{r}-\omega\right)\left\langle\omega-\left(\frac{3}{I_{1}(\overline{\mathbf{B}})}\right)^{r}\right\rangle$

With $\langle$.$\rangle is the positive part function, h$ is a characteristic time and $r$ is a parameter.

At last, the volumic part of the free energy is chosen to be written as follows:

$\psi^{v o l}(J)=\mathrm{k}(J-1)^{2}$

Where $k$ is a constant similar to a compressibility modulus.

\section{RESULTS AND DISCUSSION}

\subsection{Identification of the model parameters}

According to the experimental observations, we want to take into account time-dependent phenomena associated with several characteristic times $h_{i}$. Thus, we extend the previous model to a case with $n$ viscous branches, so that the viscous free energy becomes:

$\psi_{v}^{i s o}\left(\overline{\mathbf{B}}_{e}, \omega_{i}\right)=\sum_{i=1}^{n} G_{i}\left(\omega_{i}\right)\left(I_{1}\left(\overline{\mathbf{B}}_{e}\right)-3\right)$ 


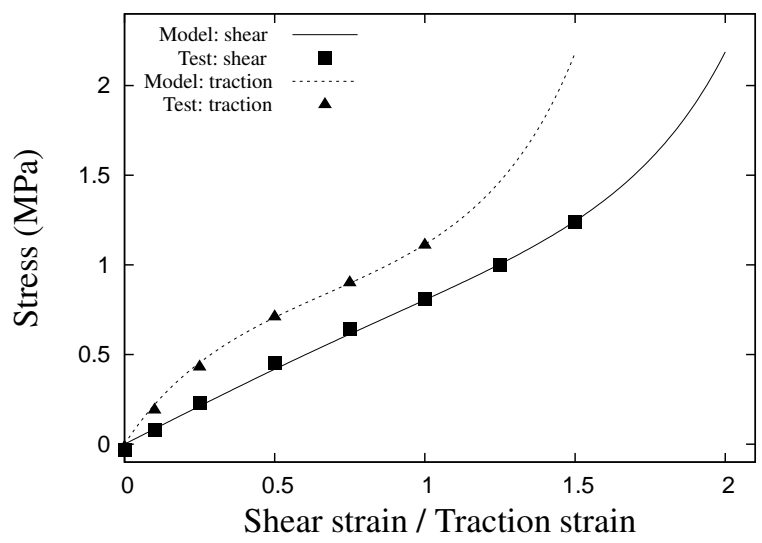

Figure 6: Identification of the elastic part of the free energy

With:

$G_{i}\left(\omega_{i}\right)=G_{i} \omega_{i}$

For each $\omega_{i}$, the evolution law is:

$\dot{\omega}_{i}=\frac{1}{h_{i}}\left(\left(\frac{3}{I_{1}(\overline{\mathbf{B}})}\right)^{r_{i}}-\omega_{i}\right)\left\langle\omega_{i}-\left(\frac{3}{I_{1}(\overline{\mathbf{B}})}\right)^{r_{i}}\right\rangle$

In order to identify the parameters of the model, we minimize the error function between the tests and the model. To do so, we use a numerical non-linear global optimization based on an genetic algorithm proposed in Mathematica.

We proceed in two steps:

1. Identification of the coefficients of the elastic part of the free energy, using the step-relaxation tests, in shear load and traction load simultaneously

2. Identification of the coefficients of the viscous part of the free energy, using traction relaxation tests and cyclic shear tests at 3 frequencies and 3 amplitudes.

The results of the identification of the elastic branch of the model and the relaxation end points in shear and tensile load are presented Fig.6. The model describes the non-linearities for the two loading modes.

Before identifying the viscous part, we have chosen to set 5 dissipative branches with 5 characteristic times defined as $\tau_{i}=\frac{2 \eta}{G_{i} \omega_{i}}$ distributed according to a logarithmic scale $(0.01 s ; 0.1 s ; 1 s ; 10 s$ and $100 s)$. The Fig.7 presents some results of the identification of the viscous branches. We can note that the model properly describes the softening behavior when amplitude increases.

\subsection{Discussion}

We now compare our model (called "Zener with Payne") with the models described in (Linder,

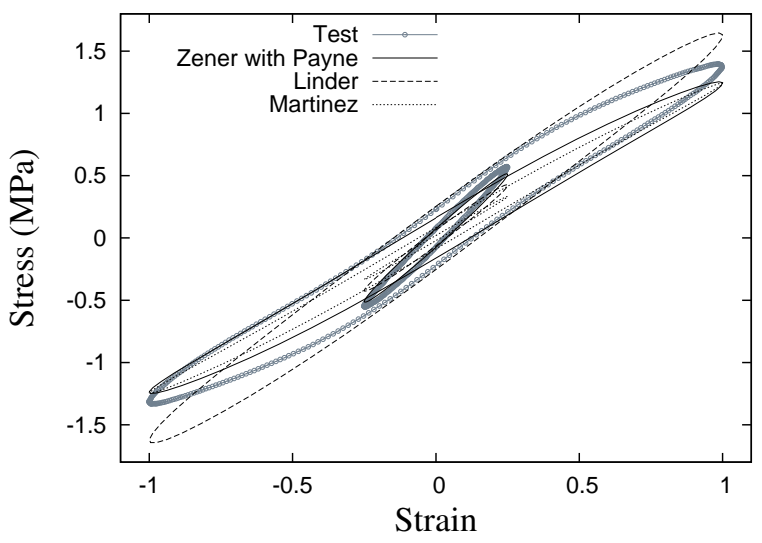

Figure 7: Stabilized cycle on a shear test at $3 \mathrm{~Hz}$ and two amplitudes $(25 \%$ and $100 \%)$

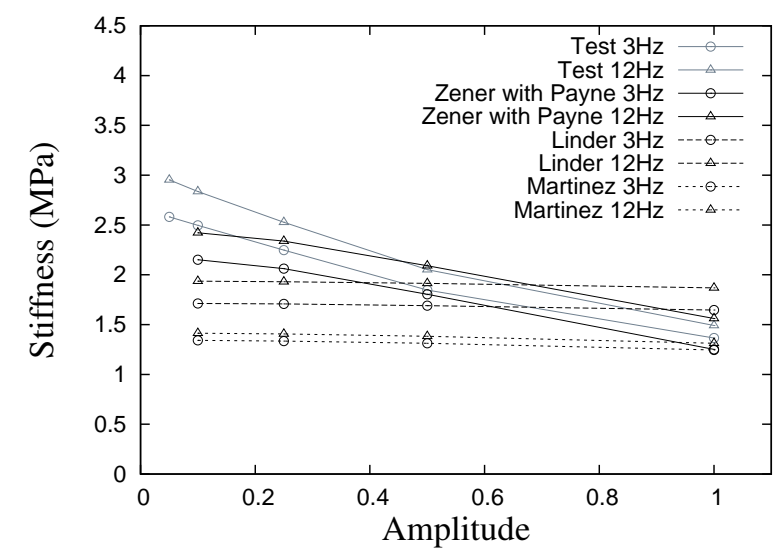

Figure 8: Effect of the amplitude on the stiffness for the different models and comparison with experiments

Tkachuk, \& Miehe 2011) and in (Martinez, Boukamel, Méo, \& Lejeunes 2011). The graphs Fig.7 and Fig.8 exhibit the Payne effect. Neither the Martinez model nor the Linder model presents an amplitude dependence. Our model correctly predict the fact that the stiffness decreases with high amplitudes whatever is the frequency. However, the stiffness remains quite underestimated at the lowest amplitudes.

Futhermore, the "Zener with Payne" model and the Linder model seem to be somehow equivalent with one another concerning the frequency-dependence of the dissipation, as shown in Fig.9. But, only our model presents the right monotony of this dependence.

If we simulate a traction test with the three models, we can see Fig.10 that our model and the Linder model present a stiffness very closed to the experiment, the third model (not represented) is not appropriate in this loading case. Moreover, our model slightly underestimates the dissipation per cycle whereas the Linder model too much dissipative.

\section{CONCLUSION}

In this study, a phenomenological model is presented. This is a multi-branch generalization of the Zener model in which internal variables have been included. 


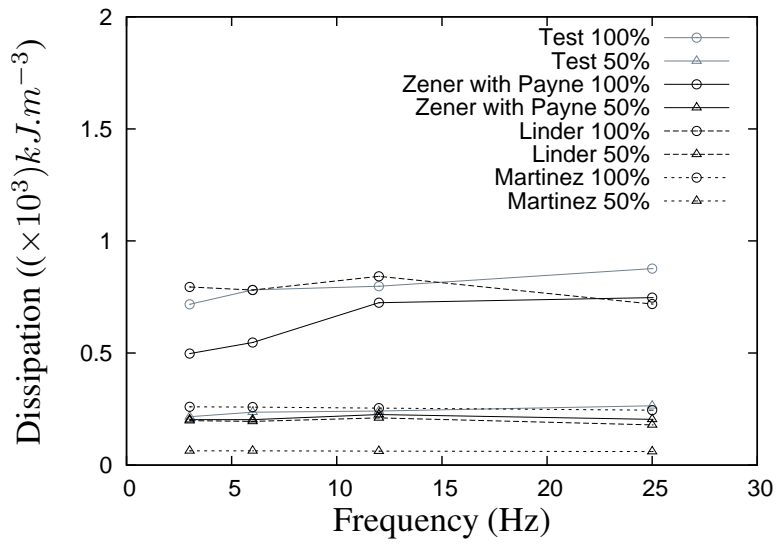

Figure 9: Effect of the frequency on the dissipation for the different models and comparison with experiments

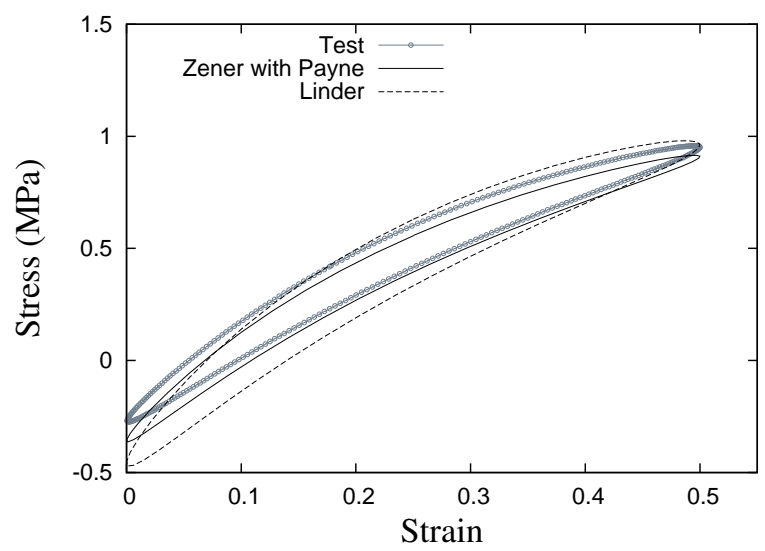

Figure 10: Stabilized cycle on a traction test at $3 \mathrm{~Hz}$ (predeformation: $25 \%$; amplitude: $25 \%$ )

Those internal variables represent time-dependent effects at the origin of the Payne effect.

The model is wanted to reproduce the influence of the frequency and the strain amplitude on the stiffness and the dissipation. Moreover, it must be valid for multi-axial loadings. The model is though identified on static and cyclic tests in both shear and traction.

The identified model is then compared with two other models: the first one is the model developped in (Martinez, Boukamel, Méo, \& Lejeunes 2011), the second one is the Linder model. The Martinez model, developped to model a silicone elastomer behavior, seem not to be suitable to describe all the phenomena experimentally observed on our material. The Linder model neither can match all the effects we want to take into account, and more specifically the Payne effect.

Moreover, the model presented in this paper has the advantage to include only scalar internal variables, contrary to the Linder model in which the internal variables are second-order tensors. In a computational point of view, this can constitute a non negligible gain.

The results show that the model properly reproduces the several phenomena observed in the range of frequencies and amplitudes eligible for our applications. Now it must be validated for multi-axial loadings. Tests will be carried out on a laminated device submitted to a combination of shear and compression load. In addition, the model will be extended to a wide range of temperature.

\section{REFERENCES}

Coleman, B. \& W. Noll (1961). Fundation of linear viscoelasticity. Rev.Mod.Phys. 33, 239-249.

Gornet, L., G. Macrkmann, R. Desmorat, \& P. Charrier (2012). A new isotropic hyperelastic strain energy function in terms of invairants and its derivation into a pseudo-elastic-model for mullins effect: Application to finite element analysis. In Constitutive Models for Rubbers VII, pp. 265-271.

Heinrich, G. \& M. Klüppel (2002). Recent advances in the theory of filler networking in elastomers. Advances in Polymer Science 160, 1-44.

Höfer, P. \& A. Lion (2009). Modelling oof frequency- and amplitude-dependent material properties of filler-reinforced rubber. J.Mech.Phys.Sol., 500-520.

Linder, C., M. Tkachuk, \& C. Miehe (2011). A micromechanicallly motivated diffusion-based transient network model and its incorporation into finite rubber viscoelasticity. J.Mech.Phys.Sol., 2134-2156.

Martinez, J. (2005). Modélisation et caractérisation du comportement hyper-visco-plastique d'un élastomère sous sollicitations multi-harmoniques et à différentes températures. $\mathrm{Ph}$. D. thesis, Univ. Aix-Marseille II.

Martinez, J., A. Boukamel, S. Méo, \& S. Lejeunes (2011). Statistical approach for a hyper-visco-plastic model for filled rubber : experimental characterization and numerical modeling. European Journal of Mechanics - A/Solids 30, 1028-1039.

Méo, S. (2000). Modélisation numérique du comportement mécanique de structures en élastomère: de l'élasticité à la thermo-visco-élasticité. Ph. D. thesis, Univ. Aix-Marseille II.

Miehe, C. \& S. Göktepe (2005). A micro-macro approach to rubber-like materials. part ii: The micro-sphere model of finite rubber viscoelasticity. J.Mech.Phys.Sol. 53, 2231-2258.

Sidoroff, F. (1974). Un modèle visoélastique non-linéaire avec configuration intermédiaire. J.Mech., 679-713.

Sidoroff, F. (1975). Variables internes en viscoélasticité, 2. milieux avec configuration intermédiaire. J.Mech., 571-595. 